\title{
Hydrogen Atom Transfer-Driven Enantioselective Minisci Reaction of Amides
}

\author{
Rupert S. J. Proctor, Padon Chuentragool, Avene C. Colgan, and Robert J. Phipps* \\ Cite This: J. Am. Chem. Soc. 2021, 143, 4928-4934 \\ Read Online
}

ABSTRACT: Minisci-type reactions constitute one of the most powerful methods for building up complexity around basic heteroarenes. The most desirable variants involve formal oxidative coupling of a $\mathrm{C}-\mathrm{H}$ bond on each partner, leading back to the simplest possible starting materials. We herein disclose a method that enables such a coupling of linear amides and heteroarenes with full control of enantioselectivity at the newly formed stereocenter as well as site selectivity on both the heteroarene and the amide. This is achieved by the use of a chiral phosphoric acid catalyst in conjunction with diacetyl as a combined hydrogen atom transfer reagent and oxidant. Diacetyl is directly photoexcitable, and thus, no extraneous photocatalyst is required: an added feature that contributes to the simplicity and practicality of the protocol.

$\mathrm{M}$ ethods for the selective conversion of $\mathrm{C}-\mathrm{H}$ bonds to new functional groups are in great demand due to the efficiency and diversity that they can impart, with vast progress being made through a variety of mechanisms. Within this broad area is a subclass of reactions that couple two components together via functionalization of a $\mathrm{C}-\mathrm{H}$ bond on each partner. In such a coupling, the complexity increase arising in a single chemical step is enormous, as the lack of a requirement for existing functional groups on each partner means they are often trivial to access. Often referred to as oxidative coupling or cross-dehydrogenative coupling reactions, these processes encompass a great breadth of mechanistic diversity, the common feature being that the formal loss of hydrogen necessitates an oxidant. ${ }^{1-12}$ In many cases a stereocenter is created, and exerting control over its formation represents an appealing way to increase the value added by the methodology even further. However, it is difficult enough to engineer a single reaction system to permit the coupling of two $\mathrm{C}-\mathrm{H}$ bonds, to superimpose a catalytic strategy for the control of enantioselectivity represents a daunting challenge. ${ }^{13-17}$ A significant proportion of enantioselective variants developed thus far is based on in situ generated iminium ions and carbocations being trapped with nucleophiles. ${ }^{18-27}$ Though further types have been reported, for example, processes proceeding via oxo-carbenium ions, ${ }^{28,29}$ metal-catalyzed cross-coupling of (hetero)arenes to form biaryls, ${ }^{30-34}$ and others, ${ }^{35,36}$ there is still a paucity of enantioselective methods considering the synthetic attractiveness afforded by the formal coupling of two $\mathrm{C}-\mathrm{H}$ bonds.

Minisci-type reactions have become one of the leading methods for heteroarene functionalization. ${ }^{37-42}$ While there are now myriad protocols for radical generation in Minisci-type reactions, those based on hydrogen atom transfer (HAT) represent a particular type of cross-dehydrogenative coupling reaction. Numerous examples have been reported, typically involving HAT from the $\alpha$-position of ethers but also increasingly from simpler alkanes. ${ }^{41,43-48}$ Recently, we developed a strategy for controlling enantioselectivity when a prochiral radical bearing an acetamido group participates in a Minisci-type reaction. ${ }^{49}$ The hypothesis was that, following protonative activation of the heteroarene by a chiral phosphoric acid (CPA), the chiral conjugate anion remains associated after radical addition, enabling enantiocontrol to be exerted in deprotonation of the resulting radical cation intermediate (Figure 1a). Computational modeling supported this hypothesis and revealed an unexpected internal mode of deprotonation (as shown). ${ }^{50}$ In our original work, we utilized redox-active esters (RAEs) derived from $N$-acetyl amino acids as radical precursors, the reduction of which formed $N$-acyl, $\alpha$ amino radicals (Figure $1 \mathrm{~b}$, upper box) ${ }^{51,52}$ In addition to providing excellent control of enantioselectivity, the CPA catalyst was able to impart high regioselectivity for the $\mathrm{C} 2$ position of the heteroarenes, whereas typically mixtures of regioisomers would be expected in many cases. ${ }^{53,54}$ Further related developments have since been made by ourselves and others. ${ }^{55-57}$ However, there are significant drawbacks to our original protocol. While the RAEs constituted very effective radical precursors, they required synthesis from the corresponding $\mathrm{N}$-acetyl amino acid, which was often low-yielding. More problematically, the number of readily commercially available amino acids is largely restricted to those that are naturally occurring and a protracted synthesis is required for most others. A further practical limitation was that some amino acid-derived RAEs exhibited poor stability upon purification and/or storage. In considering these drawbacks, we speculated whether it may be possible to combine HAT-driven radical

Received: February 8, 2021

Published: March 29, 2021 
a Outline of key steps in CPA-catalyzed asymmetric Minisci reaction
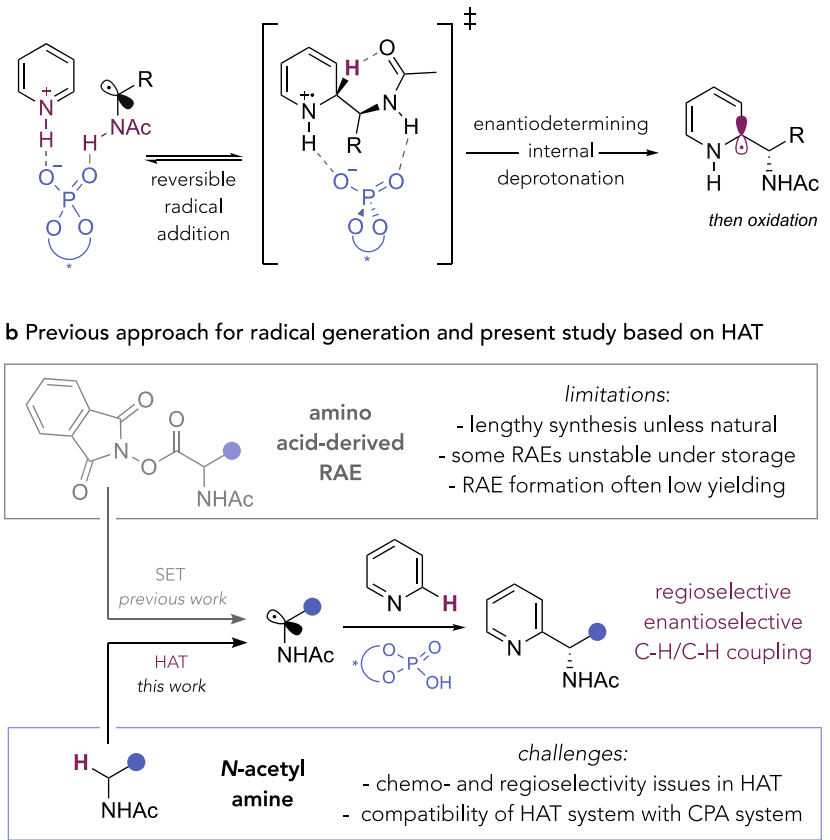

Figure 1. Background to the CPA-catalyzed Minisci reaction and the aims of this study.

formation with our CPA-catalyzed strategy for the control of selectivity. Such an approach would obviate the need for prefunctionalization of the radical precursor, constituting a formal coupling of two $\mathrm{C}-\mathrm{H}$ bonds with control over both enantioselectivity and regioselectivity in the product (Figure $1 b$, lower box).

Several major obstacles must be overcome in order to realize this goal. While Minisci-type reactions involving HAT from ethers are numerous and typically facile, those involving HAT from amides to generate $\alpha$-amino radicals are far fewer. ${ }^{58-68}$ Furthermore, it is notable that most of these involve amides derived from secondary amines, which bear no $\mathrm{N}-\mathrm{H}$ on the resulting radical. These are unlikely to be conducive to selectivity in our protocol, as experimental and computational studies have firmly established that the $\mathrm{N}-\mathrm{H}$ functionality plays a crucial role in interacting with the catalyst (Figure 1a). ${ }^{50}$ Only a handful of HAT-driven Minisci reaction reports contain examples of successful HAT from the $\alpha$-position of $N$ acylated primary amines, causing concern that this may be problematic. ${ }^{61,62}$ We anticipated that a major challenge would be to achieve this in the presence of other abstractable hydrogen atoms. Encouragingly, a number of recent studies involving nickel catalysis have achieved selective HAT from the $\alpha$-position of secondary amides in which the bromine radical is implicated as HAT reagent. ${ }^{69,70}$ Other important studies have demonstrated site-selective HAT from the $\alpha$-position of secondary carbamates. ${ }^{71,72}$ This gave us optimism that a selective HAT reagent may be identifiable. A potential complication in our case is that a stoichiometric oxidant must be compatible with the HAT system; ideally, a single reagent would perform both functions.

We commenced our studies with lepidine (1) and $N$ acetylphenethylamine (2). The latter is a challenging radical precursor due to the possibility for HAT to occur from the benzylic position. However, as part of our optimization, we particularly sought to identify a HAT protocol that would be selective for the position $\alpha$ to amines even in the presence of other weak $\mathrm{C}-\mathrm{H}$ bonds. ${ }^{72}$ We initially examined peroxides as a combined HAT reagent source and oxidant. In the presence of a photocatalyst and irradiation with blue LEDs, we envisaged that photosensitized peroxide cleavage may occur (Table 1). ${ }^{73,74}$ Although 1,4-dioxane had been the optimal solvent

Table 1. Optimization of the HAT-Driven Enantioselective Minisci Reaction $^{a}$
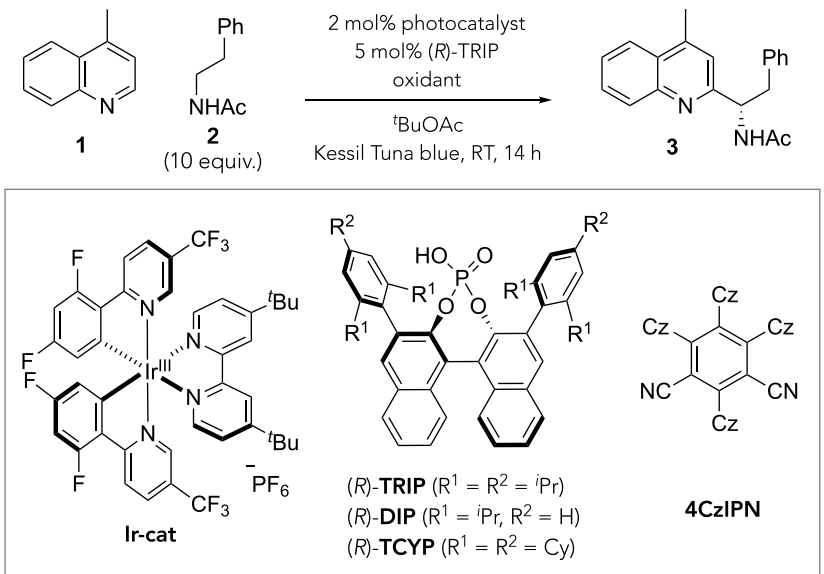

$\begin{array}{lclccc}\text { entry } & \text { photocatalyst } & \text { oxidant/equiv. } & \text { solvent } & \text { yield } \% & \text { ee/\% } \\ 1 & \text { Ir-cat } & \left({ }^{t} \mathrm{BuO}\right)_{2}(3) & { }^{t} \mathrm{BuOAc} & 15 & 96 \\ 2 & 4 \mathrm{CzIPN} & \left({ }^{t} \mathrm{BuO}\right)_{2}(3) & { }^{t} \mathrm{BuOAc} & 13 & 96 \\ 3 & 4 \mathrm{CzIPN} & \mathrm{DCP}(3) & { }^{t} \mathrm{BuOAc} & 7 & 92 \\ 4 & 4 \mathrm{CzIPN} & (\mathrm{BzO})_{2}(3) & { }^{t} \mathrm{BuOAc} & <5 & \text { nd } \\ 5 & \text { none } & \left(\mathrm{CH}_{3} \mathrm{CO}\right)_{2}(25) & { }^{t} \mathrm{BuOAc} & 77 & 93 \\ 6 & \text { none } & \left(\mathrm{CH}_{3} \mathrm{CO}\right)_{2}(10) & { }^{t} \mathrm{BuOAc} & 85 & 93 \\ 7 & \text { none } & \left(\mathrm{CH}_{3} \mathrm{CO}\right)_{2}(5) & { }^{t} \mathrm{BuOAc} & 85 & 93 \\ 8^{b} & \text { none } & \left(\mathrm{CH}_{3} \mathrm{CO}\right)_{2}(10) & { }^{t} \mathrm{BuOAc} & 66 & 94 \\ 9 & \text { none } & \left(\mathrm{CH}_{3} \mathrm{CO}\right)_{2}(10) & \text { EtOAc } & 65 & 93 \\ 10^{c} & \text { none } & \left(\mathrm{CH}_{3} \mathrm{CO}\right)_{2}(10) & { }^{t} \mathrm{BuOAc} & 0 & \end{array}$

${ }^{a}$ Yield determined by ${ }^{1} \mathrm{H}$ NMR with reference to 1,3,5-trimethoxybenzene. ee determined by SFC. ${ }^{b} 5$ equiv of amide. ${ }^{c}$ Reaction run in the dark.

previously, its liability to undergo HAT prompted us to switch to ${ }^{t} \mathrm{BuOAc}$. Encouraging initial results were obtained using ditert-butyl peroxide (DTBP), $\operatorname{Ir}\left[\mathrm{dF}\left(\mathrm{CF}_{3}\right) \mathrm{ppy}\right]_{2}(\mathrm{dtbpy}) \mathrm{PF}_{6}(\mathrm{Ir}-$ cat) as photocatalyst, and (R)-TRIP as the CPA (entry 1). While the product yield was low (15\%), we were pleased to observe that the enantiomeric excess was excellent (96\%), suggesting that the crucial parts of the CPA cycle were not being disrupted by the HAT process. We next evaluated the organic dye photocatalyst 4CzIPN and obtained comparable results (entry 2). Whereas dicumyl peroxide (DCP) gave similar results to DTBP (entry 3), dibenzoyl peroxide gave no discernable product (entry 4). Although the enantiomeric excesses were excellent, we were unable to increase the chemical yields to levels $>20 \%$ using peroxides as oxidants, despite extensive efforts. In most cases, mass balance was poor, and we concluded that the peroxide was inducing multiple decomposition pathways, possibly via nonselective HAT or overoxidation of various intermediates. In attempting to overcome this, our attention was drawn to an interesting recent study from $\mathrm{Li}$ and co-workers, in which HAT-induced Minisci reactions from ethers are carried out simply by visible light irradiation of diacetyl $\left(\left(\mathrm{CH}_{3} \mathrm{CO}\right)_{2}\right)$ in the presence of 
acid. ${ }^{75}$ Diacetyl is a cheap, low molecular weight oxidant that absorbs in the $380-460 \mathrm{~nm}$ region, and $\mathrm{Li}$ and co-workers' report demonstrated that it is competent at performing HAT on certain ethers possessing easily cleavable $\alpha-\mathrm{C}-\mathrm{H}$ bonds. A particularly attractive feature is that no added photocatalyst is required due to diacetyl's ability to be directly excited using visible light. Replacing the peroxide and photocatalyst with 25 equiv of diacetyl, according to $\mathrm{Li}$ and co-workers' protocol, we were very happy to observe that a yield of $77 \%$ could be obtained with little loss of enantioselectivity (entry 5). We next found that the equivalents of diacetyl could be reduced to ten (entry 6) and in this case even five (entry 7); although for the scope exploration, we preferred to use 10 equivalents, as for some less reactive substrates this was found to be superior. The use of an equimolar amount of benzil in place of an excess of diacetyl was not viable (see the Supporting Information). ${ }^{75}$ The reduction of the equivalents of amide to five led to an unacceptable drop in yield (entry 8) as did the use of EtOAc as solvent (entry 9). The performance of the optimal reaction in the dark led to no product formation, suggesting that photoexcitation of diacetyl is crucial (entry 10).

We first evaluated the substrate scope of $\mathrm{N}$-acetylated primary amines (Scheme 1). As well as simple $N$ acetylphenethylamine (3), a number of other phenethylamine-derived amides were effective. Fluoro- (4) and

\section{Scheme 1. Scope of $N$-Acetylated Primary Amines ${ }^{a}$}

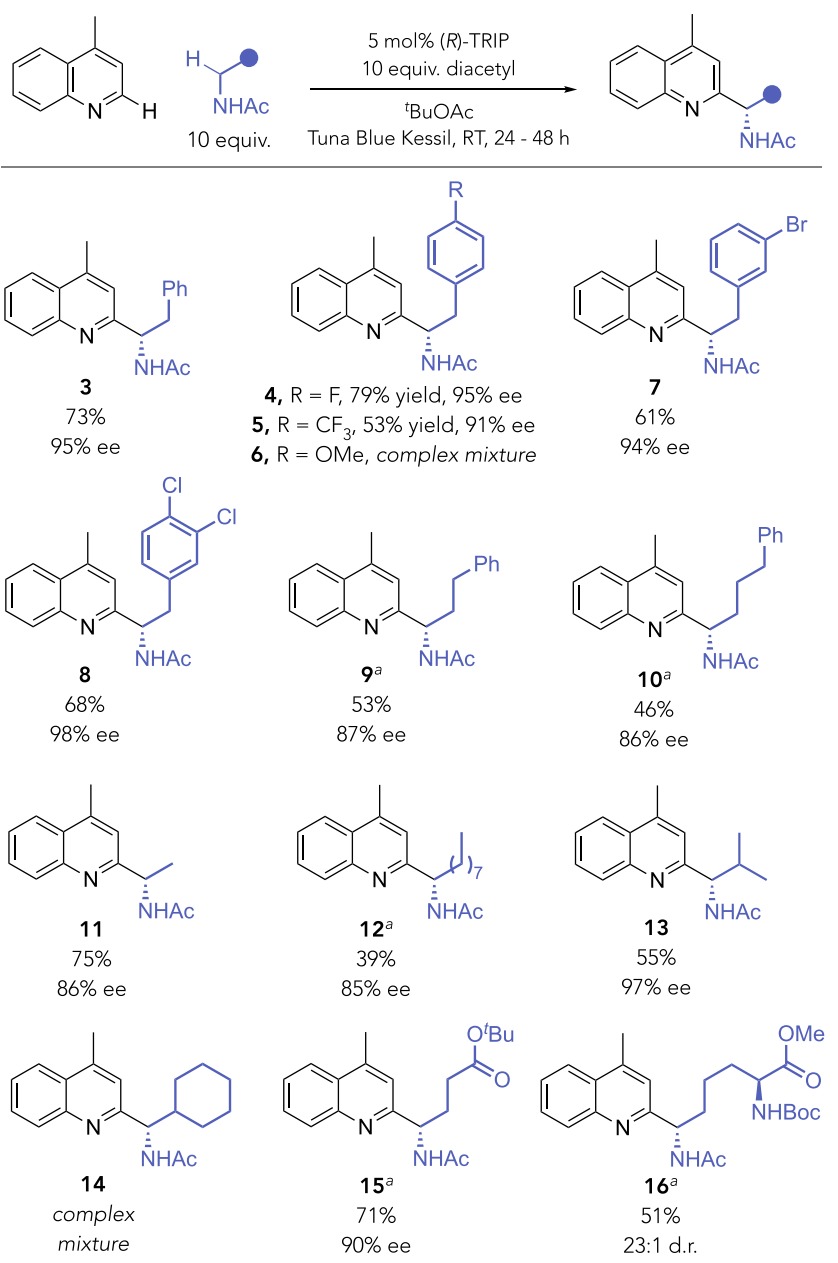

${ }^{a}$ Using (R)-DIP as catalyst. trifluoromethyl (5) substituents could be smoothly incorporated into the arene para position, although a methoxy group at this position gave a complex mixture, possibly a result of competing HAT at the benzylic position (6). A bromo substituent at the meta position was accommodated with no side reactions occurring at the bromine (7), and a 3,4-dichloro substitution pattern was equally well tolerated (8). It is important to note that the synthesis of RAE precursors for these products via the amino acid would be lengthy, whereas the acetylamine can be simply prepared by acetylation of the phenethylamine or in two easy steps from the corresponding nitrile. We found that homologated amides with the phenyl ring one (9) and two (10) methylene units further away than in phenethylamine still function as effective radical precursors and the presence of these more distant benzylic hydrogen atoms was not problematic. The removal of the aromatic ring was tolerated: simple $\mathrm{N}$-acetylethylamine performed very well, giving both good yield and ee (11), and a related longer alkyl chain amide was also effective (12). We were pleased to discover that, under our optimized conditions, HAT could be carried out selectively adjacent to the amide even in the presence of a weak tertiary alkyl $\mathrm{C}-\mathrm{H}$ bond (13) with the product formed in excellent enantiomeric excess. That said, we did find that exchanging the isopropyl group for a cyclohexyl group resulted in a complex mixture, suggesting that excessive HAT from the cycloalkane portion may have been occurring in this case (14). A remote ester could be smoothly incorporated into the amide precursor (15) and an enantiopure lysinederived amide, featuring two differentially protected amines, gave the product with a high diastereomeric ratio (16). For the amides with longer chain lengths, the CPA $(R)$-DIP, in which the $i \operatorname{Pr}$ groups at the 4 and $4^{\prime}$ positions are removed, was found to provide optimal enantioselectivity $(9,10,12,15,16)$. The remaining mass balance of the heteroarene was mostly accounted for by the starting material in all cases, and any moderate yields are not due to the formation of isomeric products. We investigated whether the addition of a photocatalyst could improve a moderate yielding example (10), but this was found not to be effective. Additionally, we have successfully carried out the reaction to give 3 on a $1 \mathrm{mmol}$ scale with no detriment to yield or ee (see the Supporting Information for details).

We next examined the scope of the heteroarene reaction component (Scheme 2). Using $\mathrm{N}$-acetylphenethylamine, we evaluated a number of quinolines. When simple quinoline, bearing no substituent at the 4-position, was used as a substrate, $>20: 1$ regioselectivity was obtained for the reaction at the 2-position (17). This result is in line with our original study and demonstrates that the extremely high levels of regiocontrol imparted by the CPA are maintained in this new HAT-driven protocol. Various functionality is tolerated at the 6-position of the quinoline including methoxy (18) and chloro (19) as well as a methyl at the 3-position (20) with no adverse effect on regioselectivity or enantioselectivity. Phenanthridine (21) and a 4-aryloxy quinoline (22) were also effective. A range of pyridines was explored in addition, with an electronwithdrawing substituent required in order to obtain reactivity. Nicotinic acid esters reacted well with methyl groups at the 2-, 4-, and 5-positions tolerated (23-26). Versatile ketone (27) and nitrile (28) functionalities were also incorporated smoothly. For the pyridines, the bulkier CPA ( $R$ )-TCYP was found to give the highest enantioselectivity. ${ }^{76}$ 
Scheme 2. Scope of Quinolines and Pyridines ${ }^{a}$
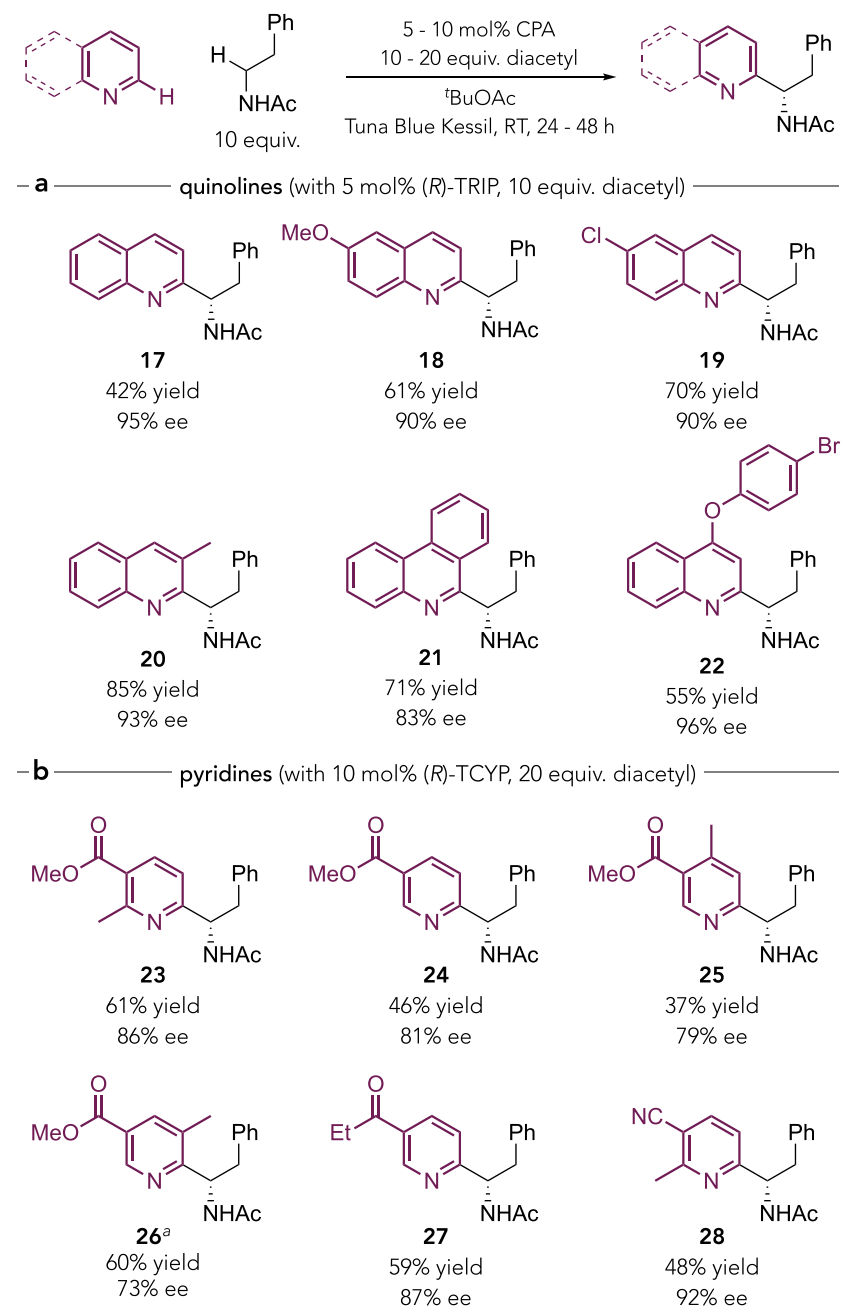

${ }^{a}$ With (R)-TRIP.

In a prior study, we found diazines to also be effective substrates for the asymmetric Minisci reaction using RAEs. However, they typically exhibited rather lower reactivity than quinolines and pyridines. ${ }^{56}$ Nevertheless, we were encouraged to find that 2-methyl-4-phenylpyrimidine successfully underwent the Minisci reaction with excellent enantioselectivity using the HAT-driven protocol (Scheme 3a). While the yield was relatively low under the present conditions, we are optimistic that future refinements may be able to improve on

\section{Scheme 3. Miscellaneous Experiments}

a Viability of a pyrimidine substrate:

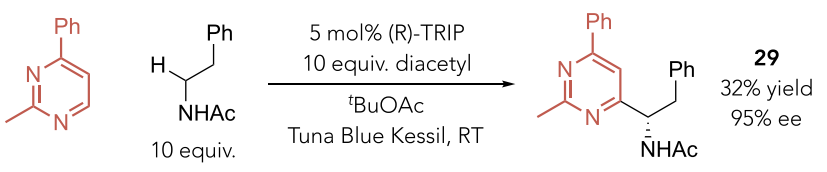

b Deprotection of the $\mathrm{N}$-acetyl group without loss of stereochemical integrity:

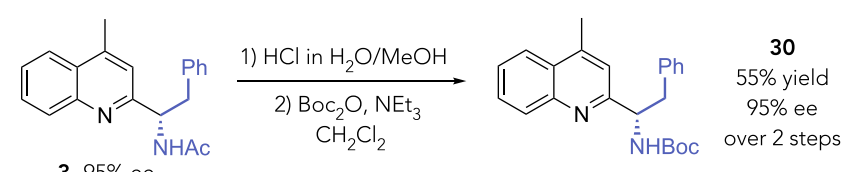

this and expand the scope of the process further. We have also demonstrated that the $\mathrm{N}$-acetyl group of a representative product can be readily deprotected under acidic conditions without the loss of stereochemical integrity (Scheme 3b).

Mechanistically, we anticipate that the photoexcited diacetyl is operating in a similar manner to that proposed by $\mathrm{Li}$ and coworkers. ${ }^{75}$ Once photoexcited, the diacetyl initiates HAT from the $N$-acetyl amine (31) (Scheme 4). The $\alpha$-amino radical

\section{Scheme 4. Plausible Mechanistic Scenario}

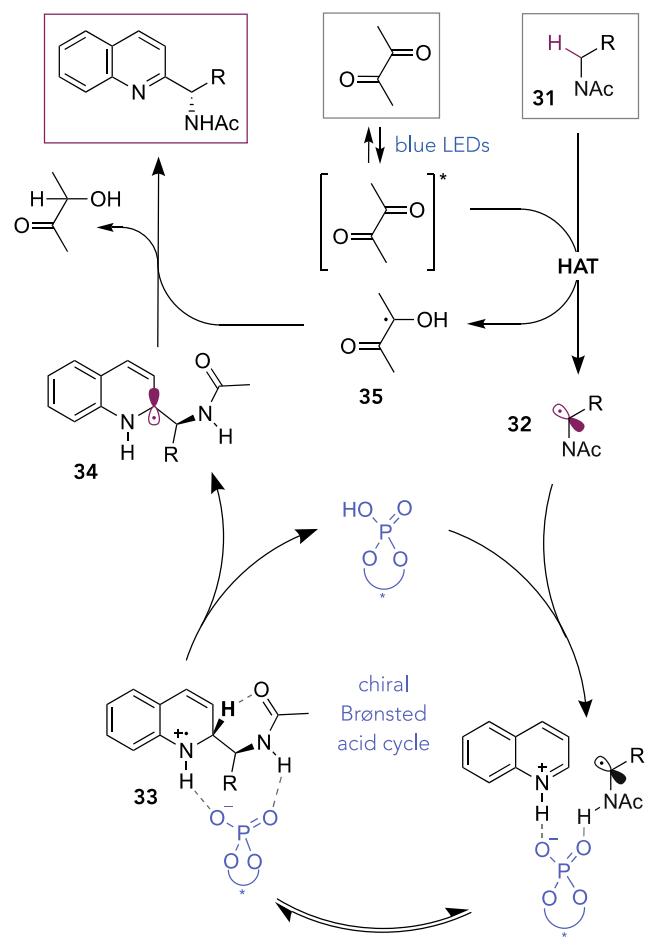

produced (32) then takes part in CPA-catalyzed radical addition, which is reversible, as determined previously. ${ }^{49,50}$ Computational studies demonstrated that deprotonation of the resulting radical cation $\mathbf{3 3}$ is the stereodetermining step and occurs through an internal deprotonation mode effected by the amide carbonyl. ${ }^{50}$ This proton is rapidly transferred to a new molecule of starting material (not shown) so that the CPA cycle can continue. In accordance with $\mathrm{Li}$ and coworkers' mechanistic evidence, we suspect that the resultant neutral radical 34 is then oxidized by ketyl radical 35 with an accompanied proton transfer, giving the Minisci product together with acetoin as the byproduct.

In summary, we have realized an enantioselective Minisci reaction that proceeds through the formal coupling of two C$\mathrm{H}$ bonds via a successful combination of HAT-driven radical generation and a CPA-catalyzed Minisci reaction. The catalyst controlled both regioselectivity and enantioselectivity. Crucial to the success was the identification of diacetyl as a mild, chemoselective reagent for the generation of $\alpha$-aminoalkyl radicals, which precluded the need for an added photocatalyst. This protocol builds up a remarkable amount of complexity in a single chemical step using very simple reaction conditions and should be directly relevant to the synthesis of small molecules of medicinal interest. 


\section{ASSOCIATED CONTENT}

\section{(s) Supporting Information}

The Supporting Information is available free of charge at https://pubs.acs.org/doi/10.1021/jacs.1c01556.

Experimental procedures and spectral data (PDF)

\section{AUTHOR INFORMATION}

\section{Corresponding Author}

Robert J. Phipps - Yusuf Hamied Department of Chemistry, University of Cambridge, Cambridge CB2 1EW, United Kingdom; (1) orcid.org/0000-0002-7383-5469; Email: rjp71@cam.ac.uk

\section{Authors}

Rupert S. J. Proctor - Yusuf Hamied Department of Chemistry, University of Cambridge, Cambridge CB2 1EW, United Kingdom; (1) orcid.org/0000-0002-2296-448X

Padon Chuentragool - Yusuf Hamied Department of Chemistry, University of Cambridge, Cambridge CB2 1EW, United Kingdom

Avene C. Colgan - Yusuf Hamied Department of Chemistry, University of Cambridge, Cambridge CB2 1EW, United Kingdom; (1) orcid.org/0000-0003-3842-6077

Complete contact information is available at: https://pubs.acs.org/10.1021/jacs.1c01556

\section{Notes}

The authors declare no competing financial interest.

\section{ACKNOWLEDGMENTS}

We are grateful to the Royal Society for a University Research Fellowship (R.J.P.), the EPSRC and GSK for a CASE studentship (R.S.J.P.), the European Research Council through Horizon 2020 (ERC Starting Grant NonCovRegioSiteCat, 757381) and the EPSRC (EP/S03269X/1).

\section{REFERENCES}

(1) Li, C.-J. Cross-Dehydrogenative Coupling (CDC): Exploring CC Bond Formations beyond Functional Group Transformations. Acc. Chem. Res. 2009, 42, 335-344.

(2) Scheuermann, C. J. Beyond Traditional Cross Couplings: The Scope of the Cross Dehydrogenative Coupling Reaction. Chem. Asian J. 2010, 5, 436-451.

(3) Yeung, C. S.; Dong, V. M. Catalytic Dehydrogenative CrossCoupling: Forming Carbon-Carbon Bonds by Oxidizing Two Carbon-Hydrogen Bonds. Chem. Rev. 2011, 111, 1215-1292.

(4) Le Bras, J.; Muzart, J. Intermolecular Dehydrogenative Heck Reactions. Chem. Rev. 2011, 111, 1170-1214.

(5) Cho, S. H.; Kim, J. Y.; Kwak, J.; Chang, S. Recent advances in the transition metal-catalyzed twofold oxidative $\mathrm{C}-\mathrm{H}$ bond activation strategy for C-C and C-N bond formation. Chem. Soc. Rev. 2011, 40, 5068-5083.

(6) Liu, C.; Zhang, H.; Shi, W.; Lei, A. Bond Formations between Two Nucleophiles: Transition Metal Catalyzed Oxidative CrossCoupling Reactions. Chem. Rev. 2011, 111, 1780-1824.

(7) Girard, S. A.; Knauber, T.; Li, C.-J. The Cross-Dehydrogenative Coupling of C-H Bonds: A Versatile Strategy for C-C Bond Formations. Angew. Chem., Int. Ed. 2014, 53, 74-100.

(8) Liu, C.; Yuan, J.; Gao, M.; Tang, S.; Li, W.; Shi, R.; Lei, A. Oxidative Coupling between Two Hydrocarbons: An Update of Recent C-H Functionalizations. Chem. Rev. 2015, 115, 1213812204 .
(9) Yang, Y.; Lan, J.; You, J. Oxidative $\mathrm{C}-\mathrm{H} / \mathrm{C}-\mathrm{H}$ Coupling Reactions between Two (Hetero)arenes. Chem. Rev. 2017, 117, 8787-8863.

(10) Kozlowski, M. C. Oxidative Coupling in Complexity Building Transforms. Acc. Chem. Res. 2017, 50, 638-643.

(11) Funes-Ardoiz, I.; Maseras, F. Oxidative Coupling Mechanisms: Current State of Understanding. ACS Catal. 2018, 8, 1161-1172.

(12) Huang, C.-Y.; Kang, H.; Li, J.; Li, C.-J. En Route to Intermolecular Cross-Dehydrogenative Coupling Reactions. J. Org. Chem. 2019, 84, 12705-12721.

(13) Giri, R.; Shi, B.-F.; Engle, K. M.; Maugel, N.; Yu, J.-Q. Transition metal-catalyzed $\mathrm{C}-\mathrm{H}$ activation reactions: diastereoselectivity and enantioselectivity. Chem. Soc. Rev. 2009, 38, 3242-3272.

(14) Zheng, C.; You, S.-L. Recent development of direct asymmetric functionalization of inert $\mathrm{C}-\mathrm{H}$ bonds. RSC $A d v$. 2014, 4, 6173-6214.

(15) Newton, C. G.; Wang, S.-G.; Oliveira, C. C.; Cramer, N. Catalytic Enantioselective Transformations Involving $\mathrm{C}-\mathrm{H}$ Bond Cleavage by Transition-Metal Complexes. Chem. Rev. 2017, 117, $8908-8976$

(16) Saint-Denis, T. G.; Zhu, R.-Y.; Chen, G.; Wu, Q.-F.; Yu, J.-Q. Enantioselective $\mathrm{C}(\mathrm{sp} 3)-\mathrm{H}$ bond activation by chiral transition metal catalysts. Science 2018, 359, No. eaao4798.

(17) Faisca Phillips, A. M.; C. Guedes da Silva, M. d. F.; Pombeiro, A. J. L. New Trends in Enantioselective Cross-Dehydrogenative Coupling. Catalysts 2020, 10, 529.

(18) Li, Z.; Li, C.-J. Catalytic Enantioselective Alkynylation of Prochiral sp3 C-H Bonds Adjacent to a Nitrogen Atom. Org. Lett. 2004, 6, 4997-4999.

(19) Guo, C.; Song, J.; Luo, S.-W.; Gong, L.-Z. Enantioselective Oxidative Cross-Coupling Reaction of 3-Indolylmethyl C-H Bonds with 1,3-Dicarbonyls Using a Chiral Lewis Acid-Bonded Nucleophile to Control Stereochemistry. Angew. Chem., Int. Ed. 2010, 49, 55585562.

(20) Zhang, G.; Zhang, Y.; Wang, R. Catalytic Asymmetric Activation of a C-H Bond Adjacent to a Nitrogen Atom: A Versatile Approach to Optically Active $\alpha$-Alkyl $\alpha$-Amino Acids and C1Alkylated Tetrahydroisoquinoline Derivatives. Angew. Chem., Int. Ed. 2011, 50, 10429-10432.

(21) DiRocco, D. A.; Rovis, T. Catalytic Asymmetric $\alpha$-Acylation of Tertiary Amines Mediated by a Dual Catalysis Mode: N-Heterocyclic Carbene and Photoredox Catalysis. J. Am. Chem. Soc. 2012, 134, 8094-8097.

(22) Zhang, G.; Ma, Y.; Wang, S.; Zhang, Y.; Wang, R. Enantioselective Metal/Organo-Catalyzed Aerobic Oxidative sp3C$\mathrm{H}$ Olefination of Tertiary Amines Using Molecular Oxygen as the Sole Oxidant. J. Am. Chem. Soc. 2012, 134, 12334-12337.

(23) Zhang, J.; Tiwari, B.; Xing, C.; Chen, X.; Chi, Y. R. Enantioselective Oxidative Cross-Dehydrogenative Coupling of Tertiary Amines to Aldehydes. Angew. Chem., Int. Ed. 2012, 51, 3649-3652.

(24) Neel, A. J.; Hehn, J. P.; Tripet, P. F.; Toste, F. D. Asymmetric Cross-Dehydrogenative Coupling Enabled by the Design and Application of Chiral Triazole-Containing Phosphoric Acids. J. Am. Chem. Soc. 2013, 135, 14044-14047.

(25) Tan, Y.; Yuan, W.; Gong, L.; Meggers, E. Aerobic Asymmetric Dehydrogenative Cross-Coupling between Two C-H Groups Catalyzed by a Chiral-at-Metal Rhodium Complex. Angew. Chem., Int. Ed. 2015, 54, 13045-13048.

(26) Yang, Q.; Zhang, L.; Ye, C.; Luo, S.; Wu, L.-Z.; Tung, C.-H. Visible-Light-Promoted Asymmetric Cross-Dehydrogenative Coupling of Tertiary Amines to Ketones by Synergistic Multiple Catalysis. Angew. Chem., Int. Ed. 2017, 56, 3694-3698.

(27) Wang, Z.; Zhu, Y.; Pan, X.; Wang, G.; Liu, L. Synthesis of Chiral Triarylmethanes Bearing All-Carbon Quaternary Stereocenters: Catalytic Asymmetric Oxidative Cross-Coupling of 2,2-Diarylacetonitriles and (Hetero)arenes. Angew. Chem., Int. Ed. 2020, 59, 30533057. 
(28) Meng, Z.; Sun, S.; Yuan, H.; Lou, H.; Liu, L. Catalytic Enantioselective Oxidative Cross-Coupling of Benzylic Ethers with Aldehydes. Angew. Chem., Int. Ed. 2014, 53, 543-547.

(29) Lee, A.; Betori, R. C.; Crane, E. A.; Scheidt, K. A. An Enantioselective Cross-Dehydrogenative Coupling Catalysis Approach to Substituted Tetrahydropyrans. J. Am. Chem. Soc. 2018, 140, 6212-6216.

(30) Narute, S.; Parnes, R.; Toste, F. D.; Pappo, D. Enantioselective Oxidative Homocoupling and Cross-Coupling of 2-Naphthols Catalyzed by Chiral Iron Phosphate Complexes. J. Am. Chem. Soc. 2016, 138, 16553-16560.

(31) Gao, D.-W.; Gu, Q.; You, S.-L. An Enantioselective Oxidative $\mathrm{C}-\mathrm{H} / \mathrm{C}-\mathrm{H}$ Cross-Coupling Reaction: Highly Efficient Method To Prepare Planar Chiral Ferrocenes. J. Am. Chem. Soc. 2016, 138, 25442547.

(32) Tian, J.-M.; Wang, A.-F.; Yang, J.-S.; Zhao, X.-J.; Tu, Y.-Q.; Zhang, S.-Y.; Chen, Z.-M. Copper-Complex-Catalyzed Asymmetric Aerobic Oxidative Cross-Coupling of 2-Naphthols: Enantioselective Synthesis of 3,3'-Substituted C1-Symmetric BINOLs. Angew. Chem., Int. Ed. 2019, 58, 11023-11027.

(33) Cai, Z.-J.; Liu, C.-X.; Gu, Q.; Zheng, C.; You, S.-L. PdIICatalyzed Regio- and Enantioselective Oxidative C-H/C-H CrossCoupling Reaction between Ferrocenes and Azoles. Angew. Chem., Int. Ed. 2019, 58, 2149-2153.

(34) Wang, Q.; Zhang, W.-W.; Song, H.; Wang, J.; Zheng, C.; Gu, Q.; You, S.-L. Rhodium-Catalyzed Atroposelective Oxidative C-H/ $\mathrm{C}-\mathrm{H}$ Cross-Coupling Reaction of 1-Aryl Isoquinoline Derivatives with Electron-Rich Heteroarenes. J. Am. Chem. Soc. 2020, 142, $15678-15685$.

(35) Conrad, J. C.; Kong, J.; Laforteza, B. N.; MacMillan, D. W. C. Enantioselective $\alpha$-Arylation of Aldehydes via Organo-SOMO Catalysis. An Ortho-Selective Arylation Reaction Based on an Open-Shell Pathway. J. Am. Chem. Soc. 2009, 131, 11640-11641.

(36) Ye, L.; Tian, Y.; Meng, X.; Gu, Q.-S.; Liu, X.-Y. Enantioselective Copper(I)/Chiral Phosphoric Acid Catalyzed Intramolecular Amination of Allylic and Benzylic C-H Bonds. Angew. Chem., Int. Ed. 2020, 59, 1129-1133.

(37) Minisci, F.; Vismara, E.; Fontana, F. Recent Developments of Free-Radical Substitutions of Heteroaromatic Bases. Heterocycles 1989, 28, 489-519.

(38) Minisci, F.; Fontana, F.; Vismara, E. Substitutions by nucleophilic free radicals: A new general reaction of heteroaromatic bases. J. Heterocycl. Chem. 1990, 27, 79-96.

(39) Duncton, M. A. J. Minisci reactions: Versatile $\mathrm{CH}$ functionalizations for medicinal chemists. MedChem Comm 2011, 2, $1135-1161$

(40) Tauber, J.; Imbri, D.; Opatz, T. Radical Addition to Iminium Ions and Cationic Heterocycles. Molecules 2014, 19, 16190.

(41) Proctor, R. S. J.; Phipps, R. J. Recent Advances in Minisci-Type Reactions. Angew. Chem., Int. Ed. 2019, 58, 13666-13699.

(42) Sun, A. C.; McAtee, R. C.; McClain, E. J.; Stephenson, C. R. J. Advancements in Visible-Light-Enabled Radical C(sp)2-H Alkylation of (Hetero)arenes. Synthesis 2019, 51, 1063-1072.

(43) Antonchick, A. P.; Burgmann, L. Direct Selective Oxidative Cross-Coupling of Simple Alkanes with Heteroarenes. Angew. Chem., Int. Ed. 2013, 52, 3267-3271.

(44) Jin, J.; MacMillan, D. W. C. Direct $\alpha$-Arylation of Ethers through the Combination of Photoredox-Mediated C-H Functionalization and the Minisci Reaction. Angew. Chem., Int. Ed. 2015, 54, $1565-1569$.

(45) Okugawa, N.; Moriyama, K.; Togo, H. Introduction of Ether Groups onto Electron-Deficient Nitrogen-Containing Heteroaromatics Using Radical Chemistry under Transition-Metal-Free Conditions. Eur. J. Org. Chem. 2015, 2015, 4973-4981.

(46) Devari, S.; Shah, B. A. Visible light-promoted C-H functionalization of ethers and electron-deficient arenes. Chem. Commun. 2016, 52, 1490-1493.
(47) McCallum, T.; Jouanno, L.-A.; Cannillo, A.; Barriault, L. Persulfate-Enabled Direct C-H Alkylation of Heteroarenes with Unactivated Ethers. Synlett 2016, 27, 1282-1286.

(48) Quattrini, M. C.; Fujii, S.; Yamada, K.; Fukuyama, T.; Ravelli, D.; Fagnoni, M.; Ryu, I. Versatile cross-dehydrogenative coupling of heteroaromatics and hydrogen donors via decatungstate photocatalysis. Chem. Commun. 2017, 53, 2335-2338.

(49) Proctor, R. S. J.; Davis, H. J.; Phipps, R. J. Catalytic enantioselective Minisci-type addition to heteroarenes. Science 2018, $360,419-422$.

(50) Ermanis, K.; Colgan, A. C.; Proctor, R. S. J.; Hadrys, B. W.; Phipps, R. J.; Goodman, J. M. A Computational and Experimental Investigation of the Origin of Selectivity in the Chiral Phosphoric Acid Catalyzed Enantioselective Minisci Reaction. J. Am. Chem. Soc. 2020, 142, 21091-21101.

(51) Cheng, W.-M.; Shang, R.; Fu, Y. Photoredox/Brønsted Acid Co-Catalysis Enabling Decarboxylative Coupling of Amino Acid and Peptide Redox-Active Esters with N-Heteroarenes. ACS Catal. 2017, 7, 907-911.

(52) Cheng, W.-M.; Shang, R.; Fu, M.-C.; Fu, Y. PhotoredoxCatalysed Decarboxylative Alkylation of N-Heteroarenes with $\mathrm{N}$ (Acyloxy)phthalimides. Chem. - Eur. J. 2017, 23, 2537-2541.

(53) O'Hara, F.; Blackmond, D. G.; Baran, P. S. Radical-Based Regioselective $\mathrm{C}-\mathrm{H}$ Functionalization of Electron-Deficient Heteroarenes: Scope, Tunability, and Predictability. J. Am. Chem. Soc. 2013, $135,12122-12134$.

(54) Hadrys, B. W.; Phipps, R. J. Acid and Solvent Effects on the Regioselectivity of Minisci-Type Addition to Quinolines Using Amino Acid Derived Redox Active Esters. Synlett 2021, 32, 179-184.

(55) Liu, X.; Liu, Y.; Chai, G.; Qiao, B.; Zhao, X.; Jiang, Z. Organocatalytic Enantioselective Addition of $\alpha$-Aminoalkyl Radicals to Isoquinolines. Org. Lett. 2018, 20, 6298-6301.

(56) Reid, J. P.; Proctor, R. S. J.; Sigman, M. S.; Phipps, R. J. Predictive Multivariate Linear Regression Analysis Guides Successful Catalytic Enantioselective Minisci Reactions of Diazines. J. Am. Chem. Soc. 2019, 141, 19178-19185.

(57) Zheng, D.; Studer, A. Asymmetric Synthesis of Heterocyclic $\gamma$ Amino-Acid and Diamine Derivatives by Three-Component Radical Cascade Reactions. Angew. Chem., Int. Ed. 2019, 58, 15803-15807.

(58) Citterio, A.; Gentile, A.; Minisci, F.; Serravalle, M.; Ventura, S. Polar effects in free-radical reactions. Carbamoylation and. alpha.-Namidoalkylation of heteroaromatic bases by amides and hydroxylamine-O-sulfonic acid. J. Org. Chem. 1984, 49, 3364-3367.

(59) Minisci, F.; Giordano, C.; Vismara, E.; Levi, S.; Tortelli, V. Polar effects in free radical reactions. Induced decompositions of peroxo compounds in the substitution of heteroaromatic bases by nucleophilic radicals. J. Am. Chem. Soc. 1984, 106, 7146-7150.

(60) Wang, J.; Li, J.; Huang, J.; Zhu, Q. Transition Metal-Free Amidoalkylation of Benzothiazoles and Amidoalkylarylation of Activated Alkenes with N,N-Dialkylamides. J. Org. Chem. 2016, 81, 3017-3022.

(61) Zhang, Y.; Teuscher, K. B.; Ji, H. Direct $\alpha$-heteroarylation of amides ( $\alpha$ to nitrogen) and ethers through a benzaldehyde-mediated photoredox reaction. Chem. Sci. 2016, 7, 2111-2118.

(62) Okugawa, N.; Moriyama, K.; Togo, H. Introduction of Quinolines and Isoquinolines onto Nonactivated $\alpha-\mathrm{C}-\mathrm{H}$ Bond of Tertiary Amides through a Radical Pathway. J. Org. Chem. 2017, 82, 170-178.

(63) Bosset, C.; Beucher, H.; Bretel, G.; Pasquier, E.; Queguiner, L.; Henry, C.; Vos, A.; Edwards, J. P.; Meerpoel, L.; Berthelot, D. Minisci-Photoredox-Mediated $\alpha$-Heteroarylation of N-Protected Secondary Amines: Remarkable Selectivity of Azetidines. Org. Lett. 2018, 20, 6003-6006.

(64) Dong, J.; Xia, Q.; Lv, X.; Yan, C.; Song, H.; Liu, Y.; Wang, Q. Photoredox-Mediated Direct Cross-Dehydrogenative Coupling of Heteroarenes and Amines. Org. Lett. 2018, 20, 5661-5665.

(65) Grainger, R.; Heightman, T. D.; Ley, S. V.; Lima, F.; Johnson, C. N. Enabling synthesis in fragment-based drug discovery by 
reactivity mapping: photoredox-mediated cross-dehydrogenative heteroarylation of cyclic amines. Chem. Sci. 2019, 10, 2264-2271.

(66) Truscello, A. M.; Gambarotti, C. Revisiting the Minisci Reaction: New Mild Amidoalkylation of Benzo-Fused N-Heteroaromatic Bases under Metal-Free Conditions. Org. Process Res. Dev. 2019, 23, 1450-1457.

(67) Vijeta, A.; Reisner, E. Carbon nitride as a heterogeneous visiblelight photocatalyst for the Minisci reaction and coupling to $\mathrm{H} 2$ production. Chem. Commun. 2019, 55, 14007-14010.

(68) Quan, Y.; Lan, G.; Fan, Y.; Shi, W.; You, E.; Lin, W. MetalOrganic Layers for Synergistic Lewis Acid and Photoredox Catalysis. J. Am. Chem. Soc. 2020, 142, 1746-1751.

(69) Rand, A. W.; Yin, H.; Xu, L.; Giacoboni, J.; Martin-Montero, R.; Romano, C.; Montgomery, J.; Martin, R. Dual Catalytic Platform for Enabling sp3 $\alpha \mathrm{C}-\mathrm{H}$ Arylation and Alkylation of Benzamides. ACS Catal. 2020, 10, 4671-4676.

(70) Shu, X.; Huan, L.; Huang, Q.; Huo, H. Direct Enantioselective $\mathrm{C}(\mathrm{sp} 3)-\mathrm{H}$ Acylation for the Synthesis of $\alpha$-Amino Ketones. J. Am. Chem. Soc. 2020, 142, 19058-19064.

(71) Le, C.; Liang, Y.; Evans, R. W.; Li, X.; MacMillan, D. W. C. Selective sp3 $\mathrm{C}-\mathrm{H}$ alkylation via polarity-match-based cross-coupling. Nature 2017, 547, 79-83.

(72) Ye, J.; Kalvet, I.; Schoenebeck, F.; Rovis, T. Direct $\alpha$-alkylation of primary aliphatic amines enabled by $\mathrm{CO} 2$ and electrostatics. Nat. Chem. 2018, 10, 1037-1041.

(73) Scaiano, J. C.; Wubbels, G. G. Photosensitized dissociation of di-tert-butyl peroxide. Energy transfer to a repulsive excited state. $J$. Am. Chem. Soc. 1981, 103, 640-645.

(74) Michelin, C.; Hoffmann, N. Photosensitization and Photocatalysis-Perspectives in Organic Synthesis. ACS Catal. 2018, 8, 12046-12055.

(75) Huang, C.-Y.; Li, J.; Liu, W.; Li, C.-J. Diacetyl as a "traceless" visible light photosensitizer in metal-free cross-dehydrogenative coupling reactions. Chem. Sci. 2019, 10, 5018-5024.

(76) Rauniyar, V.; Wang, Z. J.; Burks, H. E.; Toste, F. D. Enantioselective Synthesis of Highly Substituted Furans by a Copper(II)-Catalyzed Cycloisomerization-Indole Addition Reaction. J. Am. Chem. Soc. 2011, 133, 8486-8489. 